\title{
Prospects for the Development of Alternative Hydropower in the Krasnoyarsk Territory
}

\author{
Konstantin S. Fediy*a, Sergey A. Vstovskiya, \\ Igor S. Fedorchenko ${ }^{b}$ and Evgeny A. Spirin ${ }^{a}$ \\ ${ }^{a}$ Siberian Federal University \\ Krasnoyarsk, Russian Federation \\ ${ }^{b}$ Siberian State University of Science and Technology \\ named after Academician M. F. Reshetnev \\ Krasnoyarsk, Russian Federation
}

Received 19.11.2020, received in revised form 01.02.2021, accepted 14.02.2021

\begin{abstract}
The disadvantage of hydroelectric dams is the flooding and a material breach of the ecology of the region, therefore the use of such hydroelectric power station is reasonable when no alternative method of providing the required power. In this regard, in most cases, it is advisable to use free-flow microelectric power plants.

Free-threaded micro hydropower plants constitute a power unit mounted in the flow of the river. The main difference between free-flow microelectric power plants and dams is the use of kinetic energy of the flow, rather than potential. This makes it possible to eliminate the need for earthworks and the construction of additional hydraulic structures. Free-threaded micro hydropower plants can be grouped, forming a cascade of micro hydro.

In order to increase the reliability of free-flow microelectric power plants, the design tends to use low-speed generators with rotation speeds from 140 to $650 \mathrm{rpm}$, which can significantly simplify or completely eliminate the transmission mechanism.
\end{abstract}

Keywords: free-flow microelectric power plants, dam hydroelectric power plants, synchronous machine, hydroelectric potential, optimization. 


\title{
Перспективы развития
}

\section{альтернативной гидроэнергетики \\ в Красноярском крае}

\author{
К.С. Федий ${ }^{\mathrm{a}}$, С.А. Встовский ${ }^{\mathrm{a}}$, \\ И. С. Федорченко ${ }^{\sigma}$, Е. А. Спирин ${ }^{\mathrm{a}}$ \\ ${ }^{a}$ Сибирский федеральный университет \\ Российская Федерачия, Красноярск \\ ${ }^{6}$ Сибирский государственный университет науки \\ и технологий имени академика М. Ф. Решетнева \\ Российская Федераиия, Красноярск
}

\begin{abstract}
Аннотация. В Сибирском регионе сложился большой дефицит энергетических мощностей, удовлетворять который средствами большой энергетики экономически и технически нецелесообразно. На быстрых реках Сибири эффективно применение погружных или наплавных свободнопоточных микроГЭС, не требующих затопления земель, создания плотин, отрицательно воздействующих на экологическую обстановку.

Недостатком плотинных ГЭС является затопление местности и существенное нарушение экологии региона, следовательно, применение таких ГЭС возможно при отсутствии альтернативного способа обеспечения требуемой мощности. В связи с этим в большинстве случаев целесообразно применение свободнопоточных микроГЭС.

Свободнопоточные микроГЭС представляют собой энергоагрегат, установленный в потоке реки. Основное отличие свободнопоточных микроГЭС от плотинных - использование кинетической энергии потока, а не потенциальной. Это дает возможность исключить необходимость проведения земляных работ и возведения дополнительных гидротехнических сооружений. Свободнопоточные микроГЭС могут быть объединены в группу, образуя каскадную микроГЭС.

С целью повышения надежности свободнопоточной микроГЭС при проектировании стремятся использовать низкоскоростные генераторы, имеющие частоты вращения от 140 до 650 об/мин, что позволяет существенно упростить либо полностью исключить передаточный механизм.
\end{abstract}

Ключевые слова: свободнопоточные микроГЭС, плотинные ГЭС, синхронная машина, гидроэнергетический потенциал, оптимизация.

Цитирование: Федий, К. С. Перспективы развития альтернативной гидроэнергетики в Красноярском крае / К. С. Федий, С. А. Встовский, И.С. Федорченко, Е. А. Спирин // Журн. Сиб. федер. ун-та. Техника и технологии, 2021, 14(1). С. 97-105. DOI: $10.17516 / 1999-494 X-0290$

В связи с интенсивным освоением природных ресурсов, удаленностью населенных пунктов от централизованных источников электроснабжения в Сибирском регионе сложился большой дефицит энергетических мощностей, удовлетворять который средствами большой энергетики экономически и технически нецелесообразно.

На быстрых реках Сибири эффективно применение погружных или наплавных свободнопоточных микроГЭС, не требующих затопления земель, создания плотин, отрицательно воздействующих на экологическую обстановку. Однако их широкое применение сдерживается отсутствием низкоскоростных герметичных генераторов и недостаточной развитостью теории взаимодействия турбины со свободным потоком; кроме этого, на пути широкого использования нетрадиционных возобновляемых источников энергии существует и немало серьезных

$$
-98-
$$


препятствий, прежде всего технико-экономического характера [1]. Это крайнее непостоянство большинства таких источников энергии во времени и в пространстве, малая плотность потоков энергии, с чем непосредственно связаны высокая капиталоемкость строительства и себестоимость энергии, значительная степень разного рода рисков.

Гидравлическая энергия рек обусловлена проекцией силы тяжести на направления движения потока воды, которая определяется разностью уровней воды в начале и в конце рассматриваемого участка реки. При разности уровней $H$, м на длине участка $l$, м и среднем расходе воды $Q, \mathrm{~m}^{3} / \mathrm{c}$, мощность водотока $P$, Вт составит

$$
P=\rho g Q H,
$$

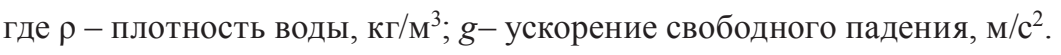

Следовательно, гидроэнергетические установки осуществляют энергетическое преобразование либо напора воды, либо водности при некоторой минимальной скорости течения.

Гидроэнергетический потенциал водотоков региона подразделяется на теоретический или валовый, технический и экономический.

Величина валовой потенциальной энергии водотока на участке реки длиной $l$, вырабатываемой за время $T$, час, равна:

$$
W=9,81 Q H T \text {. }
$$

Расход воды по длине участка непостоянен, поэтому обычно используется линейное приближение изменения расхода вдоль участка:

$$
Q_{c p}=\left(Q_{1}+Q_{2}\right) / 2
$$

где $Q_{1}, Q_{2}$ - расходы в начале и конце анализируемого участка водотока.

Таким образом, последовательно разбивая водоток на характерные участки, производим определение теоретического потенциала соответствующих участков и суммарного энергетического потенциала водотока.

Анализ гидрологических данных по рекам Сибири свидетельствует о том, что все параметры рек являются стохастическими параметрами, зависящими от большого числа факторов, таких как помесячное распределение осадков, температурный режим и мн. др. Эти параметры изменяются в широком диапазоне: скорость потока - в 2-4 раза; глубина - в 1,3-2 раза; секундный расход - до 50 раз.

Однако закон их распределения одинаков и параметры рассеяния весьма стабильны. Так, коэффициент вариации скорости колеблется от 0,11 до 0,25 , а коэффициент вариации секундного расхода - от 0,15 до 0,3. В целом можно отметить, что водотоки Сибири характеризуются очень большими сезонными изменениями своих параметров [2]. Так, гистограмма распределения секундных расходов р. Туба (рис. 1) свидетельствует о 25-кратном отличии в них. Минимум расходов приходится на январь-март, а максимум - на июнь. Эта картина типична для большинства рек края. Закон распределения расходов, близкий к нормальному.

Основная часть стоимости плотинной ГЭС состоит из стоимостей гидроагрегатов, плотины, экологического ущерба от затопления земель. Тогда стоимость плотинной ГЭС можно описать следующим образом: 


$$
C_{\Pi_{-} \Gamma \ni C}=c_{1} q_{1}+c_{2} q_{1}+c_{3} q_{3}
$$

где $c_{1}$ - стоимость 1 кВт мощности энергоблока; $q_{1}$ - количество киловатт устанавливаемой мощности; $c_{2}$ - стоимость строительства плотины, отнесенная на 1 кВт; $c_{3}$ - стоимость одного квадратного метра затопленных земель; $q_{3}$ - площадь затопления.

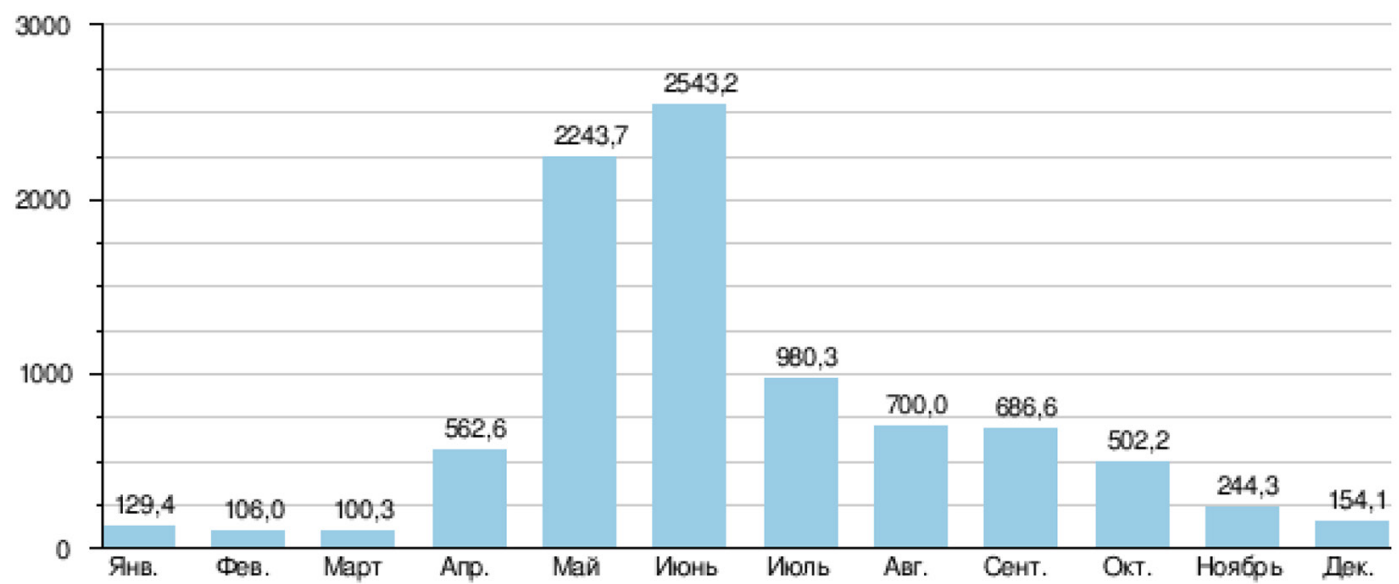

Рис. 1. Гистограмма распределения секундных расходов р. Туба

Fig. 1. Histogram of the distribution of the second flow rates of the river Tuba

Стоимость 1 кВт мощности энергоблока описывается формулой $c_{I}=f\left(q_{1}\right)$. Стоимость плотины на один киловатт мощности также можно описать в виде функции:

$$
c_{2}=f\left(q_{1}\right) k_{\text {рельефа }},
$$

где $k_{\text {рельефа }}$ - коэффициент, учитывающий рельеф местности и ее геологические особенности.

Стоимость одного квадратного метра затопленных земель также является функцией множества параметров:

$$
c_{2}=f(M),
$$

где $M$ - вектор множества параметров, зависящих от местности, на которой возводится плотина, суммарной мощности установленных агрегатов и т. п.

Площадь затопления $q_{3}$ зависит от рельефа местности, напора, расхода реки и т. д.:

$$
q_{3}=f(M) .
$$

Мы видим, что величины, влияющие на стоимость плотинной ГЭС, описаны функционалами, зависящими от множества параметров М. Для решения задачи оптимизации могут быть применены одни из методов вариационного исчисления или динамического программирования.

Так, плотинные ГЭС обычно применяют для больших мощностей, и стоимость каждой плотины очень зависит от местных условий, для эффективного применения данной методики будет нужен целый ряд сведений и материалов, характеризующих условия строительства. Эти

$$
-100-
$$


данные получают в результате изысканий и сбора необходимых материалов и сведений, освещающих следующие вопросы:

1) рельеф, растительность, существующие сооружения, населенные пункты и прочие объекты на водосборе и вблизи строительства будущей станции; эти сведения получают топографическими изысканиями;

2) режим русла и водный режим реки; эти сведения получают гидрологическими изысканиями;

3) характер грунтов и подземных вод в основании будущих сооружений, наличие местных строительных материалов; эти сведения дают геологические изыскания;

4) экономика района (наличие рабочей силы, механизмов, энергии, возможность сбыта электроэнергии, дорожная сеть и т. д.); экономические сведения собирают при экономическом обследовании района строительства.

Существенным недостатком плотинных ГЭС является затопление местности и существенное нарушение экологии региона, следовательно, применение таких ГЭС оправданно при отсутствии альтернативного способа обеспечения требуемой мощности. В связи с этим в большинстве случаев целесообразно применение свободнопоточных микроГЭС.

Свободнопоточные микроГЭС представляют собой энергоагрегат (или несколько энергоагрегатов), установленный в потоке реки. Основное отличие свободнопоточных микроГЭС от плотинных - использование кинетической энергии потока, а не потенциальной. Это дает возможность исключить необходимость проведения земляных работ и возведения дополнительных гидротехнических сооружений. Свободнопоточные микроГЭС могут быть объединены в группу, образуя каскадную микроГЭС.

Основными компонентами свободнопоточной ГЭС являются: генератор, турбина, передаточный механизм, несущая конструкция, система автоматического регулирования, защитное устройство. Следовательно, стоимость свободнопоточной ГЭС можно записать в виде формулы

$$
C_{\Pi_{-} \Gamma \ni C}=c_{1} q_{1}+c_{2} q_{2}+c_{3} q_{1}+c_{4} q_{2},
$$

где $c_{1}$ - стоимость 1 кВт мощности генератора; $q_{1}$ - количество киловатт устанавливаемой мощности; $c_{2}$ - стоимость 1 квадратного метра площади сечения турбины в плоскости нормальной к набегающему потоку (площадь сечения турбины); $q_{2}$ - площадь сечения турбины; $c_{3}$ - стоимость передаточного механизма в расчете на 1 кВт; $c_{4}$ - стоимость несущей конструкции в расчете на единицу площади сечения турбины.

Оптимизация решений, связанных с использованием свободнопоточной микроГЭС, является комплексной задачей, сочетающей в себе как оптимизацию отдельных компонентов микроГЭС, так и оптимизацию применения каскада таких установок.

С целью повышения надежности свободнопоточной микроГЭС при проектировании стремятся использовать низкоскоростные генераторы, имеющие частоты вращения от 140 до 650 об/мин, что позволяет существенно упростить либо полностью исключить передаточный механизм. Для примера рассмотрим низкоскоростные генераторы научно-исследовательского центра «ВИНДЭК», параметры которых указаны в табл. 1.

Определим зависимость стоимости 1 кВт установленной мощности генератора в зависимости от суммарной мощности генератора и частоты его вращения. Для этого построим 
Таблица 1. Параметры генераторов научно-исследовательского центра «ВИНДЭК»

Table 1. Parameters of generators of the research center "VINDEK"

\begin{tabular}{|c|c|c|c|c|c|c|c|c|c|}
\hline & 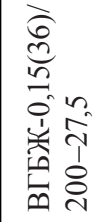 & 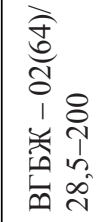 & 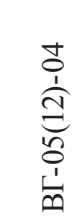 & 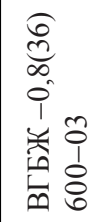 & 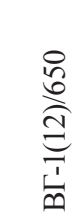 & 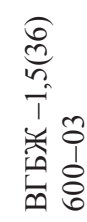 & 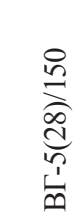 & 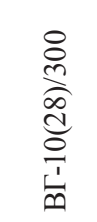 & 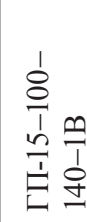 \\
\hline Мощность, Вт & 150 & 200 & 500 & 800 & 1000 & 1500 & 5000 & 10000 & 15000 \\
\hline Напряжение, В & 28,5 & 28,5 & 28,5 & 28,5 & 57 & 57 & 114 & 228 & 100 \\
\hline Частота вращения, об/мин & 200 & 200 & 650 & 600 & 450 & 600 & 150 & 300 & 140 \\
\hline КПД & 0,75 & 0,9 & 0,7 & 0,8 & 0,9 & 0,85 & 0,85 & 0,85 & 0,85 \\
\hline Macca, кг & 13 & 10 & 11 & 14 & 19 & 16 & 120 & 200 & 495 \\
\hline Стоимость, тыс. руб. & 25,2 & 25,2 & 12,6 & 30 & 14,4 & 36 & 156 & 168 & 360 \\
\hline
\end{tabular}

функции мощности генератора и его стоимости в зависимости от частоты вращения, воспользовавшись методом кусочно-линейной интерполяции данных, приведенных в табл. 1 (рис. 2, 3).

Как видно на рис. 2, 3, разреженность матрицы параметров генератора приводит к наличию высоких градиентов в аппроксимирующей функции, что может привести к неадекватным решениям задачи. При расширении параметрического ряда генераторов аппроксимирующая функция будет иметь более гладкий характер, что позволит применить градиентные методы решения задачи оптимизации.

В качестве альтернативы кусочно-линейной аппроксимации введем функцию, описывающую области применения того или иного генератора в виде спектра и состоящую из последовательности ступенчатых функций, зависящих от частоты вращения и мощности (рис. 4). Физический смысл в таком случае будут иметь все значения функции, отличные от нуля. Ширина

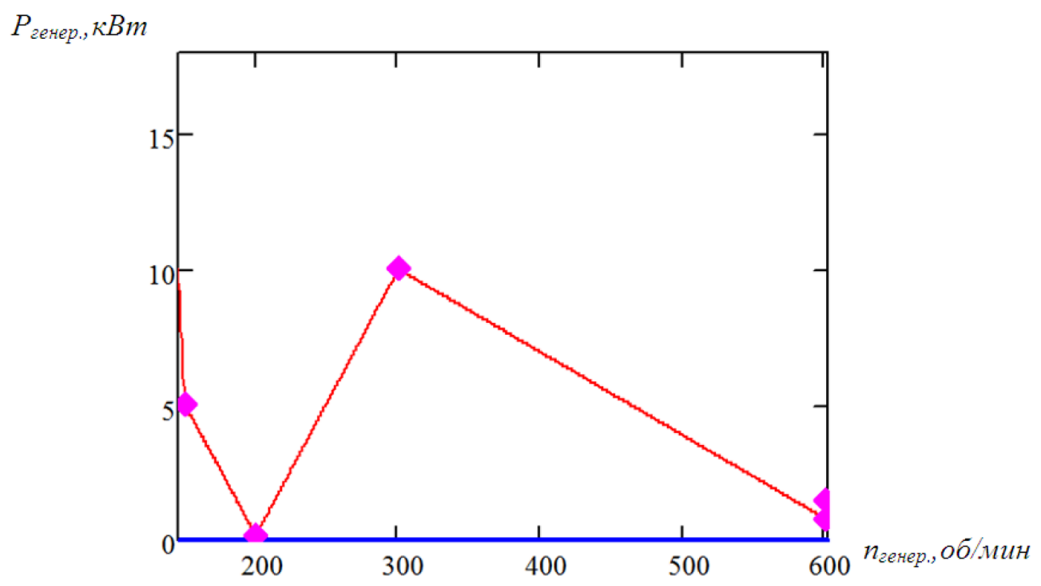

Рис. 2. Функция мощности генератора в зависимости от частоты вращения ротора

Fig. 2. Function of the generator power depending on the rotor speed 


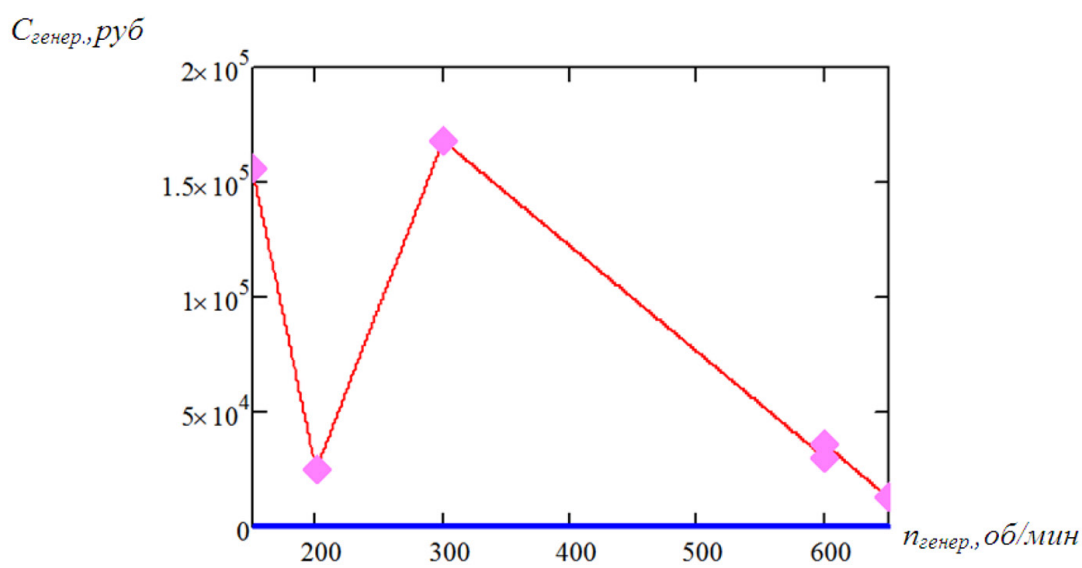

Рис. 3. Функция стоимости генератора в зависимости от частоты вращения ротора

Fig. 3. Function of the generator cost depending on the rotor speed

спектральной линии соответствует диапазонам частот вращения генератора, близким к номинальной, а амплитуда - номинальной мощности генератора [3].

Рисунок 4 иллюстрирует эффективность применения различных генераторов при помощи функций мощности генератора, полученных методом кусочно-линейной интерполяции, и спектральной функции. Кроме того, описание областей применения генераторов и их стоимости в виде спектра позволяет выполнить локализацию решений функции стоимости микроГЭС.

Аналитическое представление связи стоимости генератора с его мощностью и частотой вращения дает возможность построить и анализировать функцию зависимости стоимости генератора в расчете на 1 кВт мощности от его частоты вращения (рис. 5).

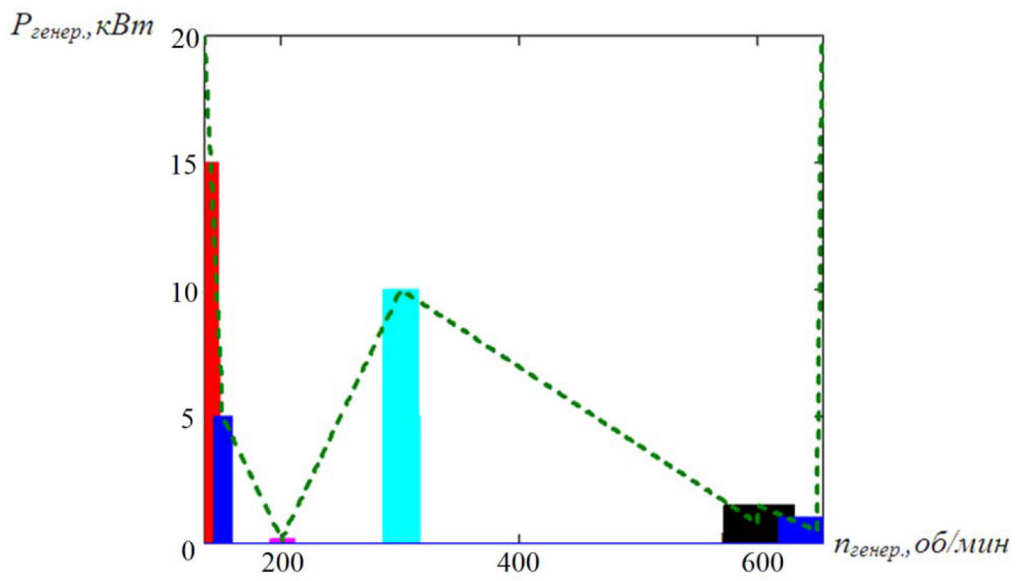

Рис. 4. Изображение функции областей применения генераторов (- - - кусочно-линейная аппроксимация мощности генератора)

Fig. 4. Image of the function of the areas of application of generators (- - piecewise-linear approximation of the generator power) 


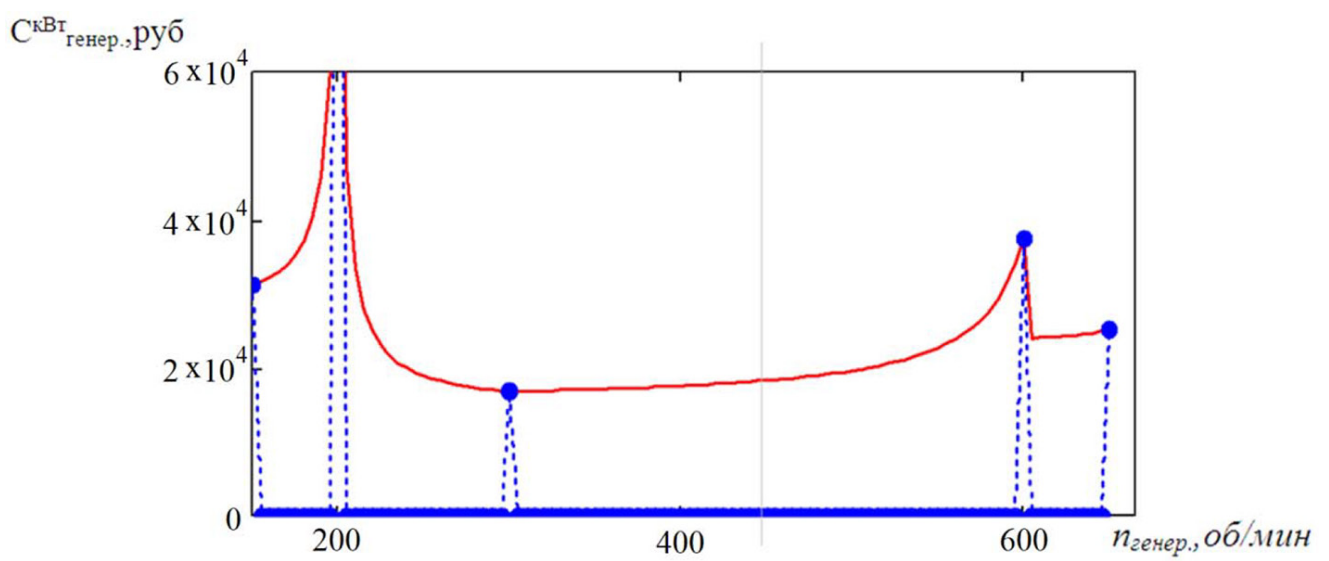

Рис. 5. Зависимость стоимости 1 кВт мощности генератора от частоты вращения ротора (- решение, получаемое при кусочно-линейной аппроксимации данных, $\bullet$ - решение, получаемое при аппроксимации данных спектральной функцией)

Fig. 5. Dependence of the cost of $1 \mathrm{~kW}$ of generator power on the rotor speed ( -- the solution obtained by piecewise linear approximation of the data, $\mathbf{0}$ - the solution obtained by approximating the data by the spectral function)

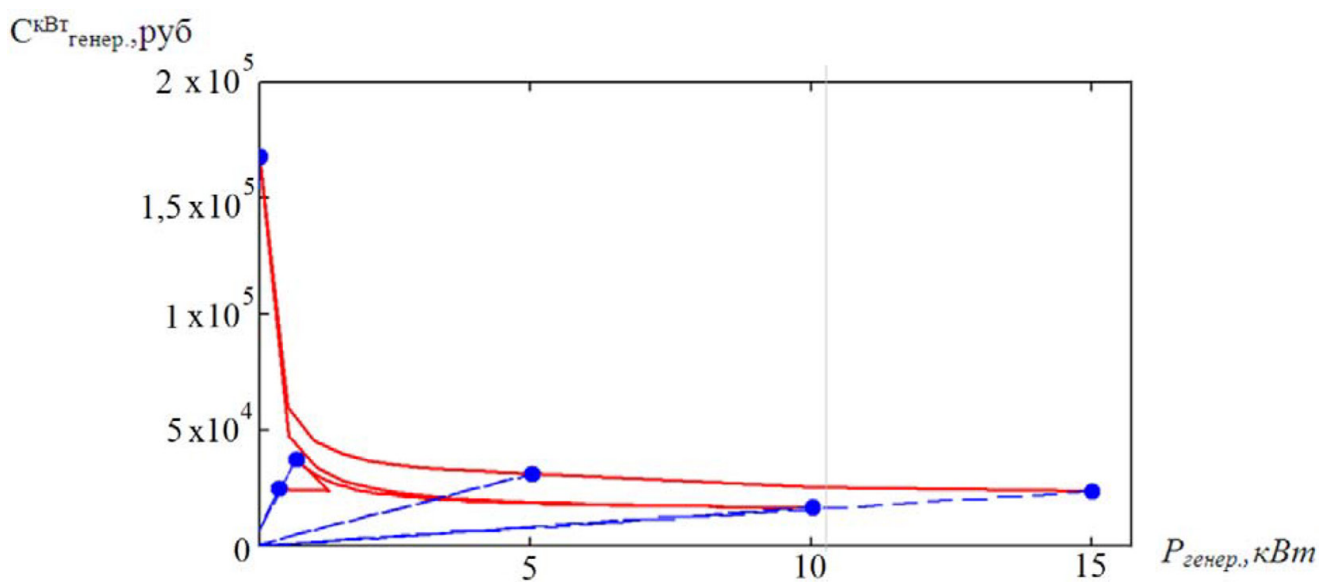

Рис. 6. Зависимость стоимости 1 кВт генератора от его мощности (- - решение, получаемое при кусочно-линейной аппроксимации данных, - - решение, получаемое при аппроксимации данных спектральной функцией)

Fig. 6. Dependence of the cost of $1 \mathrm{~kW}$ of the generator on its power ( -- the solution obtained by piecewise linear approximation of the data, - the solution obtained by approximating the data with a spectral function)

Варьируя частоты вращения генератора в диапазоне от 140 до 650 об/мин, построим зависимость стоимости генератора в расчете на 1 кВт от его мощности (рис. 6).

На графике, изображенном на рис. 6, видно, что оптимальным решением с позиций стоимости является генератор мощностью 10 кВт, частота вращения ротора которого $\mathrm{n}_{\text {ген }}=300$. Формируя различные таблицы параметров генератора и используя вышеприведенную модель, можно выбрать оптимальный генератор для свободнопоточной микроГЭС в зависимости 
от требуемой частоты вращения и мощности, что обуславливает перспективность данного направления.

\section{Список литературы / References}

[1] Спирин Е.А., Головин М.П. Перспективы использования малой гидроэнергетики в Сибирском регионе. Вестник СибГАУ, Красноярск, 2010, с. 179-184. [Spirin E. A., Golovin M.P. Prospects for the use of small hydropower in the Siberian region. Bulletin of SibGAU, Krasnoyarsk, 2010, pp. 179-184 (in Russian)]

[2] Гончаров А. Н. Гидроэлектрическое оборудование гидроэлектростанций и его монтаж. М.: Энергия, 1974. 305 c. [Goncharov A. N. Hydroelectric equipment of hydroelectric power plants and its installation. M., Energy, 1974. 305 p. (in Russian)]

[3] Безруких П.П. [и др.]. Ресурсы и эффективность использования возобновляемых источников энергии в России. СПб.: Наука, 2002. 314 с. [Bezrukih P. P. [and others]. Resources and efficiency of the use of renewable energy sources in Russia. SPb., Science, 2002. 314 p. (in Russian)] 